\title{
REALIDAD SOCIOECONÓMICA DE LOS JÓVENES MIGRANTES DEL MUNICIPIO DE SAN MIGUEL.
}

\section{SOCIOECONOMIC REALITY OF MIGRANT YOUTH FROM THE MUNICIPALITY OF SAN MIGUEL}

\author{
Pedro Adalberto Castro Cruz | Sociólogo \\ José Rigoberto Vaquerano Benavides | Master en Administración de Empresas \\ Investigadores | Universidad de Oriente \\ pcastro@univo.edu.sv | jvaquerano@univo.edu.sv
}

\section{Resumen}

La migración irregular ha sido un fenómeno sociohistórico persistente en los últimos cuarenta años en El Salvador, motivado fundamentalmente por las difíciles condiciones económicas y la falta de oportunidades laborales. En el país, se generan escenarios de exclusión social que impulsan la emigración de muchos ciudadanos que se marchan con la esperanza de obtener prosperidad económica y un entorno sociocultural estable en otros territorios. Siendo un fenómeno de gran trascendencia, la migración irregular debe ser abordada desde una perspectiva multicausal y científica, enfocando sus matices económicos, políticos y socioculturales, en los que se ve involucrada mayoritariamente la población joven. La aproximación a dicho fenómeno permite redefinir acciones de gobiernos e instituciones de la sociedad en favor de la integridad, la dignidad y el respeto a los derechos humanos de las personas migrantes y retornadas, lo que permite implementar estrategias interinstitucionales en materia socioeducativa y laboral que incidan en la prevención de la migración. Partiendo de estas consideraciones, se investigó la realidad de jóvenes estudiantes del municipio de San Miguel residentes en zonas urbanas y rurales, para conocer sus expectativas y opiniones respecto a la posibilidad de migrar debido a las condiciones socioeconómicas y al contexto de inseguridad en que viven; asimismo, se estudiaron las experiencias de jóvenes que vivieron de cerca el fenómeno de la migración irregular y volvieron al país en calidad de retornados. Por otra parte, se indagó en el trabajo de asistencia de diversas instituciones que se vinculan medularmente con migrantes y retornados, mediante iniciativas y proyectos para su reinserción socioeconómica.

Palabras clave: Migración irregular, jóvenes, realidad socioeconómica, El Salvador.

\section{Abstract}

Irregular migration has been a persistent sociohistorical phenomenon during the last forty years in El Salvador, mainly driven by the economic difficulties and the lack of job 
opportunities. The social exclusion scenarios generated in the country propel the emigration of many citizens that go abroad with the hope of finding economic prosperity and a stable sociocultural environment in other territories.

Being irregular immigration an important phenomenon, it has to be addressed from a scientific and multi-causal perspective, focusing on the economic, political and sociocultural aspects in which the young population is mainly involved.

Approximation to this phenomenon allows the redefinition of social institutions and government policies, favoring the integrity, dignity and respect for the human rights of migrant and returnees, which allows the implementation of interinstitutional strategies in labor and socio-educational aspects that have an impact on migration prevention.

The reality of rural and urban student residents from the municipality of San Miguel was studied to identify their expectations regarding the possibility of migrating, according to the socioeconomic conditions and the insecurity context in which they live in; additionally, the experience of the youth that underwent irregular migration and then came back to the country was studied as well.

Finally, the work of different aid institutions linked mainly with migrants and returnees throughout initiatives and reintegration projects was investigated.

Keywords: Irregular migration, youth, socioeconomic reality, El Salvador.

\section{Introducción}

El país fue testigo de un flujo migratorio importante de salvadoreños hacia Honduras en busca de trabajo y tierras para cultivo desde 1920 (Gobierno de El Salvador, 2017), el cual se interrumpió en 1969; este terminó abruptamente con el conflicto bélico con dicho país, que había sido la "válvula de escape demográfico" más inmediata de que se disponía. Adicionalmente, Honduras decidió cerrar su frontera por once años a los productos salvadoreños y acabó con el Mercado Común Centroamericano (Villalobos, 2017), lo que afectó el crecimiento económico y agudizó la crisis por la carencia de empleo.

Posteriormente, los salvadoreños provenientes de las zonas urbanas y rurales debido a la estrechez del territorio, la falta de oportunidades laborales y el creciente clima de violencia política previo a la guerra civil de los años ochenta encuentran un nuevo destino: Los Estados Unidos de Norteamérica. Nace así lo que luego se denominará como "el sueño americano". Este país se convierte en otra vía de escape para gran parte de la población salvadoreña que emigra hacia "el norte" del continente americano, buscando salir de una estructura 
económica, política y social débil que no responde a sus necesidades, por lo que el flujo migratorio se incrementa durante y posteriormente a los años de guerra civil.

En El Salvador, el 88.8 \% del total de migrantes están entre las edades de 18 y 65 años. La búsqueda de mejores condiciones de vida e ingresos económicos son los principales motivos por los que salvadoreños deciden abandonar el país y emigrar hacia los Estados Unidos (Corado, 2016). Existen otras causales, como deseos de reunificación familiar, inseguridad y violencia intrafamiliar. Lo anterior no significa que en muchos casos no confluya más de un motivo; de ahí la importancia de destacar la multicausalidad de la migración (Gobierno de El Salvador, 2017).

Como lo señalan estudios previos, "la migración irregular es percibida, desde el punto de vista de los Estados, como un fenómeno social que hay que regular por medio de leyes, es decir, como un problema de legalidad. Los Estados negocian entre sí políticas relativas a la migración que permitan mantener acuerdos geopolíticos de mayor dimensión, entre ellos los relativos a la seguridad y a macroagendas económicas. Por su lado, los migrantes abordan el tema de la migración desde la perspectiva del derecho humano a la alimentación, al trabajo, a una vida digna y a la reunificación familiar para la consecución de oportunidades que conduzcan a ella" (Gaborit et al., 20I2).

El enfoque teórico de esta investigación enfatiza en las causas económicas y sociales que le dan origen, basándose en la explicación que ofrece la Escuela Neoclásica sobre la migración internacional, la cual plantea que "el origen de la migración internacional es de carácter económico y han de buscarse sus causas en la diferencia salarial entre países, viéndose reflejados en el nivel de ingresos como también en las disparidades de bienestar social. La decisión de emigrar está basada en la llamada 'elección racional', con el objetivo de maximizar una función de utilidad con unos determinados rendimientos netos esperados".

Para esta escuela la decisión de emigrar está basada en la llamada, "elección racional”, con el objetivo de maximizar una función de utilidad con unos determinados rendimientos netos esperados; el movimiento migratorio es, entonces, la manifestación de la movilidad de factores para hacer el mejor uso alternativo de estos, en donde en el plano macro se le considera una teoría de la llamada redistribución espacial de los factores de producción en respuesta a precios relativos (Gómez Walteros, 2010).

\section{Metodología}

La investigación comprendió un diseño mixto. En un primer momento fue de carácter 
descriptiva y explicativa porque se propuso especificar propiedades, características y rasgos importantes del fenómeno, describiendo y analizando tendencias de un grupo o población (Hernández Sampieri et al., 20l4).

La fase cuantitativa del estudio estuvo constituida por 300 jóvenes estudiantes de 8 instituciones de educación básica y media de los sectores urbanos y rurales de la ciudad de San Miguel, obtenida a través de un muestreo probabilístico estratificado, utilizando la fórmula de poblaciones finitas.

Posteriormente, se implementó un enfoque cualitativo dirigido fundamentalmente a obtener un conocimiento más profundo de los procesos y reconstrucción del significado, con procedimientos especialmente inductivos y una orientación holística.

Se realizaron 8 entrevistas semiestructuradas a interlocutores (5 personas retornadas y 3 representantes institucionales) mediante muestreo intencionado o dirigido. Este tipo de muestreo también se clasifica como 'no probabilístico' y la elección de sus elementos no depende de la probabilidad, sino de causas relacionadas con las características de la investigación (Hernández Sampieri et al., 2014)

\section{Resultados}

Estos se subdividieron, según el abordaje de la investigación, en tres segmentos:

\section{a) Jóvenes estudiantes de educación media}

Como se aprecia en la figura I, se identificaron las actividades principales de los estudiantes, las cuales comprendían en su mayoría el desempeño académico de tiempo completo, mientras que los que estudian y trabajan evidencian un valor significativo equivalente a 82 estudiantes de los diferentes centros educativos.

Figura I. Actividad principal del estudiante

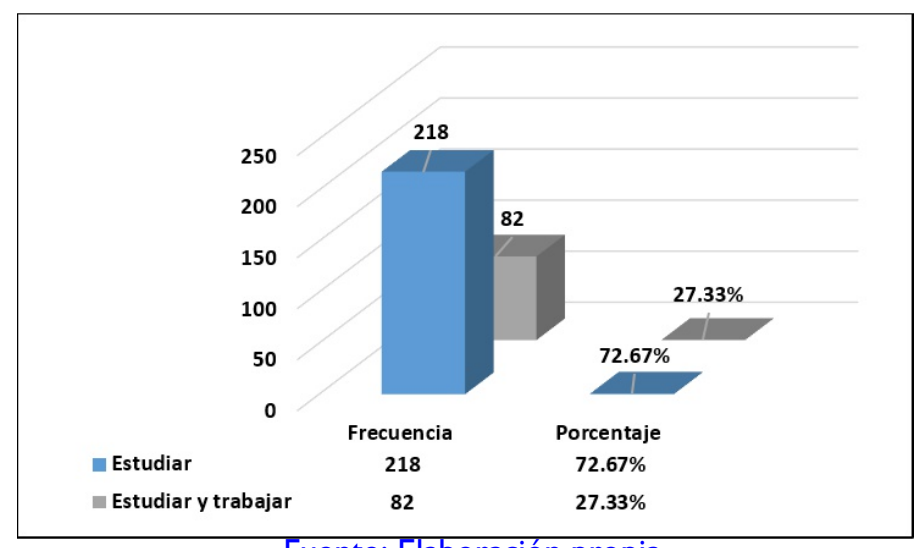

Fuente: Elaboración propia 
En la figura 2, podemos observar una frecuencia significativa que responde a una mayoría de la muestra que posee familiares en el exterior como parte del fenómeno migratorio; no así una minoría que, sin embargo, es consciente del fenómeno.

Figura 2. Núcleo familiar con miembros que han emigrado

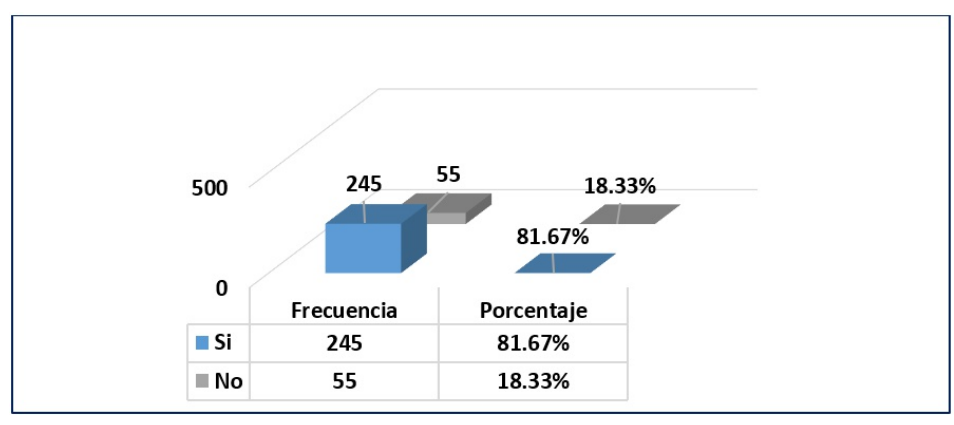

Fuente: Elaboración propia

Sin duda las razones económicas siguen siendo una constante en las iniciativas por las cuales las personas migran considerando las pocas oportunidades de empleo que brinda el país; a esto se le suma una variante como la inseguridad, la cual se ha ido al alza en los últimos años, producto de la falta de políticas de seguridad eficaces para el combate a la delincuencia. Sin embargo, también existen personas que tienen la esperanza de reencontrarse con sus familiares que han migrado.

Figura 3: Motivos por los que su familiar emigró

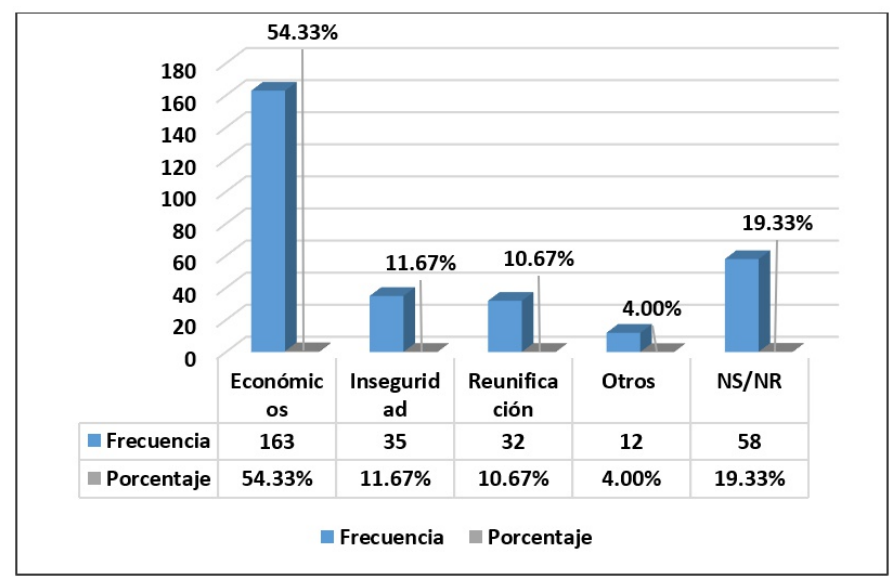

Fuente: Elaboración propia

Desde que la población salvadoreña comenzó a migrar, la recepción de remesas ha sido un elemento clave para la dinamización de la economía de las familias, generando un poder adquisitivo mayor. En la actualidad, esto sigue siendo una tendencia, ya que casi dos tercios de la muestra encuestada perciben ingresos por remesas de sus familiares. 
Figura 4. Recepción de remesas en el núcleo familiar

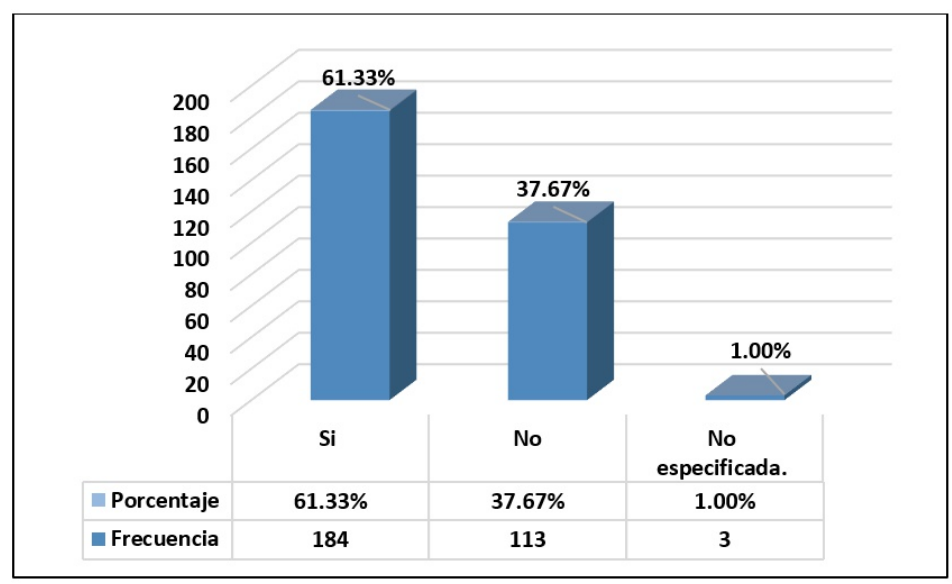

Fuente: Elaboración propia

En cuanto al uso que se le da a la remesa, la priorización de las necesidades básicas en la familia se ve reflejada al responder la mayoría que estas son utilizadas para proveer alimentación a sus hogares, seguido de un esfuerzo por formar académicamente a sus hijos $y$, en menor grado, la búsqueda de una salud plena. Es evidente que no existe una cultura de ahorro por parte de algunas familias, sin generalizar, debido a que esto depende de las condiciones materiales de vida y el nivel de ingresos del núcleo familiar.

Figura 5. Uso que la familia le da a las remesas

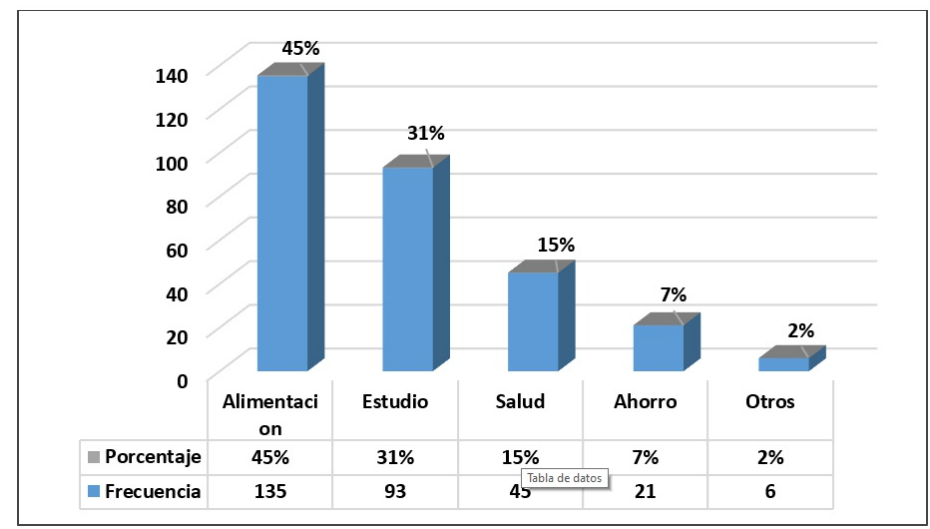

Fuente: Elaboración propia

La evaluación que hacen los jóvenes sobre las políticas de Estado en cuanto a seguridad pública es de reprobación a las mismas, al expresar que son poco efectivas y para otros sin ningún efecto positivo. Únicamente para 2 de cada 10 jóvenes son efectivas, por lo que se evidencia que existe una imagen negativa entre los jóvenes respecto a la protección que les pueda ofrecer el Estado y muy poca confianza en sus acciones para con ellos. 
Figura 6. Políticas de Estado en seguridad pública

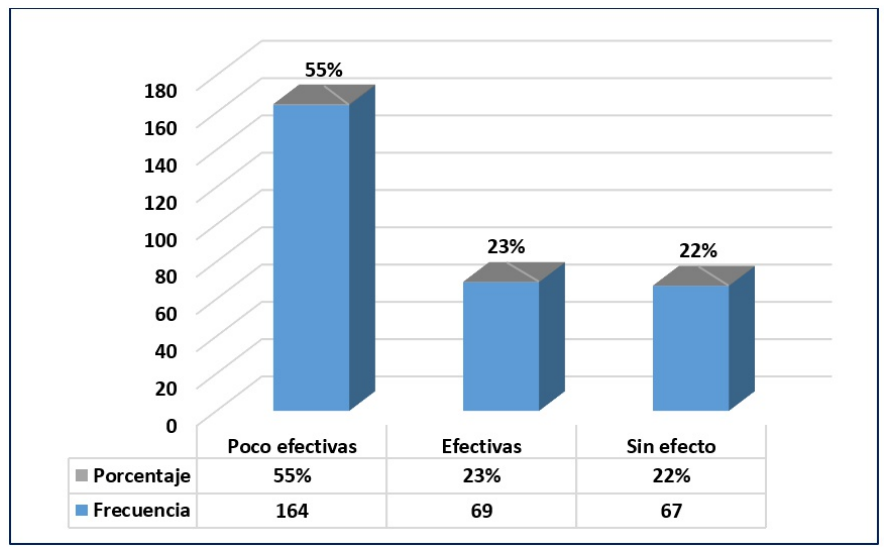

Fuente: Elaboración propia

Tabla I: Problemas de la juventud salvadoreña

\begin{tabular}{|r|r|r|r|}
\hline Problemas de la juventud en El Salvador & Frecuencia & Soluciones que se proponen & Frecuencia \\
\hline Violencia y amenazas de pandillas & 49 & Fomentar oportunidades de trabajo y talleres de \\
formación
\end{tabular}

Fuente: Elaboración propia

El mayor problema que consideran los jóvenes que afrontan son la violencia y las amenazas de las pandillas, las cuales los han convertido en un sector vulnerable de la sociedad; como segundo aspecto, el desempleo y la pobreza que los limita en sus aspiraciones de progreso económico. Es relevante mencionar también la poca atención que les dan sus padres, el peligro de caer en el consumo de las drogas y algunos de ellos aceptan que no se esfuerzan lo suficiente al sentirse desmotivados en su vida.

Como soluciones a los problemas mencionados, proponen fomentar oportunidades de trabajo y ofrecer formación en diversas especialidades laborales, realizar cambios que traigan mejoras al sistema educativo, generar los espacios para la sana recreación por medio del deporte y las artes; piden que las autoridades mejoren su trabajo de seguridad pública, hacen un llamado a otros jóvenes a que cambien actitudes negativas e improductivas, e instan a padres de familia a que les presten mayor atención a sus hijos.

\section{b) Jóvenes retornados al país}

Según lo expresado por los interlocutores, las principales causas de la migración fueron la 
búsqueda de mejores oportunidades laborales, prevaleciendo los intereses de crecer económicamente; la delincuencia ha sido la segunda razón por la cual los jóvenes tuvieron que emprender el camino.

El periodo de permanencia en territorio extranjero fue entre uno a cinco años para quienes llegaron al país de destino y laboraron en diversos trabajos, los cuales les permitió una relativa estabilidad económica durante su estancia. Parte de ese tiempo, algunos de ellos estuvieron detenidos a causa de su condición migratoria irregular. Otros migrantes fueron detenidos durante su travesía y permanecieron entre dos a tres meses en centros de detención antes de su deportación.

Entre los hechos más relevantes que narran las personas que han experimentado la migración, se mencionan: la dependencia casi absoluta de los guías de viaje, conocidos como "coyotes"; el hacinamiento de personas durante el viaje; el riesgo de ser víctimas de redes delincuenciales; la persecución y el maltrato de los agentes migratorios, y tener que soportar duras condiciones climáticas.

Los jóvenes retornados, a pesar de que han pasado por dificultares en torno a su regreso a $\mathrm{EI}$ Salvador, tienen esperanzas que descansan, en primer lugar, en la continuación de su formación académica, como es el caso de algunos de ellos estudiantes de nivel superior en calidad de becados; otros albergan expectativas de emprender un negocio y buscan financiamientos accesibles o capital semilla, y algunos desean emplearse según sus habilidades adquiridas durante su estadía en el exterior, siendo estas técnicas y cualificadas, a pesar de que no tienen un estudio medio y superior.

\section{c) Representantes institucionales}

Los representantes de las instituciones abordadas expresaron que la asistencia que ellos brindan a las personas retornadas se centra en seis aspectos:

I. Procesos de sensibilización a la población para no estigmatizar al retornado, facilitando que estos sean aceptados nuevamente en sus comunidades.

2. Capacitación e inserción laboral de retornados, ofreciéndoles certificaciones de sus habilidades laborales, pasantías, y gestionándoles empleo.

3. Aportes económicos en efectivo. En algunos casos, se les brinda la mitad de un sueldo en sus primeros tres meses de trabajo y se les da un monto en efectivo a quienes estudian.

4. Atención psicológica al retornado, debido a los traumas padecidos durante su viaje migratorio, detención y deportación. 
5. Gestión de becas de estudio para retornados, de acuerdo a sus vocaciones e interés por superarse académicamente.

6. Gestión de fondos a través de donaciones ante organismos privados y públicos y gobiernos extranjeros para financiar sus actividades y programas de atención a retornados.

Las organizaciones que trabajan con retornados tienen entre dos a cuatro años de estarles brindando atención, dos de ellas como ONG y otra es dependencia del Gobierno salvadoreño. Han establecido que el tiempo necesario para reintegrar socioeconómicamente a una persona retornada es de seis a ocho meses.

Las instituciones argumentan que es poco lo que se puede hacer para disuadir a los potenciales migrantes debido a que en algunas comunidades está fuertemente arraigado que el éxito económico solamente se puede obtener saliendo del país. Ellos hacen esfuerzos a través de charlas preventivas e informativas donde tratan de concientizar a la población de los riesgos de la migración y el accionar delictivo de algunos "coyotes" o guías de viaje. Sin embargo, reconocen que toda persona tiene derecho a migrar, aceptando que el país ofrece muy pocas oportunidades de sobrevivencia económica.

\section{Discusión}

Los resultados del presente estudio guardan estrecha relación con lo expresado por Gómez Walteros (20l0), en su artículo "La migración internacional: teorías y enfoques, una mirada actual", en el cual explica que la postura de la Escuela Neoclásica de la economía es que "el origen de la migración internacional es de carácter económico y han de buscarse sus causas en la diferencia salarial entre países, viéndose reflejados en el nivel de ingresos como también en las disparidades de bienestar social".

Este aspecto es confirmado por los jóvenes encuestados al manifestar el $81.67 \%$ de ellos que tienen familiares que migraron por causas fundamentalmente económicas. Así mismo, los cinco jóvenes retornados que se entrevistaron indicaron que se aventuraron a migrar de forma irregular por motivaciones económicas, sumado a esto la presión ejercida por el clima de violencia e inseguridad social que fue su segunda causa.

\section{Conclusiones}

" Los jóvenes no tienen un concepto muy claro sobre lo que significa la migración: algunos no pudieron definirla con precisión y otros consideran que es el desplazamiento de personas que buscan mejorar sus condiciones económicas; tienen conciencia de lo que implica realizar un 
viaje riesgoso en condiciones irregulares, muchas veces originado por la violencia y la falta de oportunidades económicas.

"Según lo expresado por los jóvenes, un aproximado de 8 de cada 10 familias tienen algún familiar que ha emigrado al extranjero desde hace más de un año, Estos lo han hecho, principalmente, por motivos económicos, seguido de la inseguridad y por reunificación familiar.

»En relación con la obtención de remesas, estas son recibidas en 6 de cada 10 hogares, frecuentemente en forma mensual, en menor medida quincenal o trimestralmente. Las familias que disponen de remesas las destinan en orden de prioridad a alimentación, educación y salud, cubriendo así sus necesidades básicas. Una baja proporción logra ahorrar parte de esos ingresos. No obstante, sostienen que las remesas no siempre cubren los gastos de educación y salud de sus hogares, debido a sus altos costos.

"En términos socioeconómicos, realizando una valoración general, se evidencia que los jóvenes viven en condiciones óptimas debido a que aún dependen de sus padres, pues por medio de ellos cubren sus necesidades básicas. En la mayoría de hogares, receptan remesas que les envían parientes en el extranjero, pocos trabajan y han crecido en un ambiente confortable; sus familias, si bien es cierto no son de altos ingresos, les han sabido suplir sus necesidades económicas y afectivas.

"Se evidencia un escenario complejo en el cual los jóvenes se desenvuelven en un contexto social excluyente que a nivel externo es hostil y amenazante para su integridad física y moral (Savenije, 2009), como también a nivel interno manifiesta episodios de conflictividad en el seno familiar y crisis individuales propias de la edad en adolescentes y jóvenes.

Los jóvenes retornados valoran su experiencia como positiva al haber adquirido competencias laborales y consideran la posibilidad de retornar al país extranjero y darles continuidad a las relaciones socioafectivas que les fueron interrumpidas. Algunos retornados no consideran emprender un nuevo viaje, dado que han encontrado oportunidades para continuar sus estudios y laborar en el país.

Las instituciones orientadas a la atención a migrantes y retornados se centralizan en su atención psicológica y económica, para luego ofrecerles capacitación laboral y becas de estudio, de tal forma que se les facilite su reinserción al aparato productivo del país.

\section{Recomendaciones}

Al Estado salvadoreño: este debe ser protagonista en los procesos de reinserción y reintegración de los compatriotas que por diversos motivos decidieron migrar y ahora han regresado voluntaria o forzosamente al suelo patrio; por ello es preciso que se visibilice el 
trabajo del Viceministerio para los Salvadoreños en el Exterior y CONMIGRANTES, organismo nacido a partir de la Ley especial para la protección y desarrollo de la persona migrante salvadoreña y su familia (Asamblea Legislativa de la República de El Salvador, 20 I I), para que la población se dé cuenta de lo que estos organismos realizan en lo concreto, sus logros y limitantes.

Al sector empresarial: abrir oportunidades de empleo a las personas retornadas para que estas fortalezcan a las empresas con la aplicación práctica de las competencias laborales adquiridas en el extranjero.

Las gremiales y fundaciones empresariales deben contribuir en la certificación de dichas competencias laborales y apoyar en el fortalecimiento de otras competencias que se requieran en el ámbito técnico laboral.

A las universidades: realizar acciones que favorezcan a la comunidad de retornados en dos vías: la primera, a través de proyección social en la lógica de la prevención del fenómeno, mediante charlas de concientización de los riesgos de la migración irregular, en instituciones educativas de educación media y superior. La segunda vía sería apoyar la formación académica del retornado mediante becas totales o parciales en alianza con instituciones estatales, municipalidades, ONG y otros.

Crear un sistema de "ventanillas únicas" de atención a personas retornadas que funcionaría como un sistema integrado en las instalaciones universitarias, posibilitándoles de esta forma una atención ágil y personalizada.

A instituciones sociales que asisten a migrantes y retornados: expandir y promocionar sus programas, ampliando la cobertura territorial a los 262 municipios del país, debido a que el fenómeno impacta en todo el territorio nacional.

Fomentar el diálogo y liderar la concreción de alianzas intersectoriales en las que se integren esfuerzos de todos los sectores de la sociedad civil en pro de los migrantes irregulares y retornados, creando zonas de producción agrícola, artesanal y servicios diversos que les permitan lograr autonomía económica a los mismos.

\section{Bibliografía}

Asamblea Legislativa de la República de El Salvador. (I4 de abril de 20II). Ley especial para la protección y desarrollo de la persona migrante salvadoreña y su familia. https:/www.asamblea.gob.sv/sites/default/files/ documents/decretos/F33C3EBE-8BBB-44 I 0-827I-005B04B6 I F0E.pdf 
Corado, I. (24 de agosto de 2016). El Salvador. Razón de la migración: ¿Economía o inseguridad? Resumen Latinoamericano. http://www.resumenlatinoamericano.org/2016/08/25/el-salvador-razon-de-la-migracioneconomia-o-inseguridad/

Gaborit, M., Zetino Duarte, M., Brioso, L. y Portillo, N. (20I2). La esperanza viaja sin visa: jóvenes y migración indocumentada en El Salvador. UNFPA-UCA. https://elsalvador.unfpa.org/sites/default/files/pub-pdf/ esperanza_sin_visa\%20\%282\%29.pdf

Gobierno de El Salvador. (2017). Política Nacional para la Protección y Desarrollo de la Persona Migrante Salvadoreña y su Familia. https://reliefweb.int/sites/reliefweb.int/files/resources/ Politica\%20Nacional\%20para\%20la\%20Proteccion\%20y\%20Desarrollo\%20de\%20la\%20Persona\%20Migran te\%20Salvadorena\%20y\%20su\%20Familia.pdf

Gómez Walteros, J. A. (2010). La migración internacional: teorías y enfoques, una mirada actual. Semestre Económico, 13 (26), 8I-99. https://www.redalyc.org/pdf/I650/I65014341004.pdf

Hernández Sampieri, R., Fernández Collado, C. y Baptista Lucio, P. (20I4). Metodología de la investigación (6.a ed.). McGraw Hill.

Savenije, W. (2009). Maras y barras. Pandillas y violencia juvenil en los barrios marginales de Centroamérica. FLACSO:Programa El Salvador. https://insumisos.com lecturasGratismaras\%20y\%20barras\%20pandillas\%20y\%20violencia\%20juvenil\%20en\%20barrios\%20margin ales\%20de\%20el\%20salvador.pdf

Villalobos, J. (I de enero de 20I7). Trump y el infierno centroamericano. Nexos. https://www.nexos.com.mx/? $\mathrm{p}=30956$ 